\title{
Assessing Malnutrition in Pregnant Women Using the Dietary Diversity Score and the Mid-Upper Arm Circumference: A Cross-Sectional Study, Zambia
}

\author{
Sebean Mayimbo', Clara Maphosa Haruzivishe², Concepta Kwaleyela ${ }^{3}$, Bwembya Phoebe ${ }^{4}$, \\ Ellen Chirwa ${ }^{5}$, Patrick Kaonga 6 , Catherine Ngoma ${ }^{1}$ \\ ${ }^{1}$ Department of Midwifery, Women and Child Health, University of Zambia, Lusaka, Zambia \\ ${ }^{2}$ Department of Nursing Sciences, University of Zimbabwe, Harare, Zimbabwe \\ ${ }^{3}$ Department of Research, Monitoring and Evaluation, University of Zambia, Lusaka, Zambia \\ ${ }^{4}$ Department of Community Health, University of Zambia, Lusaka, Zambia \\ ${ }^{5}$ Department of Midwifery, Kamuzu College of Nursing, Blantyre, Malawi \\ ${ }^{6}$ Department of Internal Medicine, Tropical Gastroenterology and Nutrition Group, University of Zambia, Lusaka, Zambia \\ Email: sebeanmayimbo@gmail.com, claraharuzivishe@yahoo.co.uk, ckwaleyela@gmail.com, pabwembya@gmail.com, \\ embweza@kcn.unima.mw, patrickkaonga@gmail.com, catherinengoma@yahoo.com
}

How to cite this paper: Mayimbo, $\mathrm{S}$, Haruzivishe, C.M., Kwaleyela, C., Phoebe, B., Chirwa, E., Kaonga, P. and Ngoma, C. (2020) Assessing Malnutrition in Pregnant Women Using the Dietary Diversity Score and the Mid-Upper Arm Circumference: A Cross-Sectional Study, Zambia. Food and Nutrition Sciences, 11, 712-725. https://doi.org/10.4236/fns.2020.117051

Received: May 30, 2020

Accepted: July 19, 2020

Published: July 22, 2020

Copyright $\odot 2020$ by author(s) and Scientific Research Publishing Inc. This work is licensed under the Creative Commons Attribution International License (CC BY 4.0).

http://creativecommons.org/licenses/by/4.0/ (c) (i) Open Access

\begin{abstract}
The dietary diversity score (DDS) and the mid-upper arm circumference (MUAC) can be used to assess nutritional status. The DDS is a qualitative measure of food consumption reflecting an individual's access to different food items and therefore a proxy indicator of the nutritional status. The aim of the study was to assess whether the dietary diversity score and MUAC can be used to assess the nutritional status of pregnant women attending antenatal. A cross-sectional survey was conducted at Chilenje level I Hospital in a township located in Lusaka city, Lusaka province. The study employed quantitative methods to collect and analyse data on the dietary patterns of individuals in the previous 24 hours before the survey. Health facilities were randomly selected and systematic sampling was used to select a sample of 299 pregnant women. An interview schedule was used to collect data. Median and interquartile range were used to compare demographic data. Spearman's Correlation for two continuous variables was used to establish associations between DDS and the MUAC. The median age was 27 years. About $44 \%$ of the women were in the lowest dietary diversity category, indicating inadequate nutrient intake, $31 \%$ in the medium category and $24 \%$ in the high intake. Dietary diversity score was negatively correlated with mid-upper arm circumference $(\mathrm{p}=0.1295)$. However, most of the women in the lower dietary
\end{abstract}


diversity score category (75\%) had a high mid-upper arm circumference meaning that their nutritional status was good. Dietary diversity score can be used to predict malnutrition in pregnant women. The mid-upper arm circumference was negatively correlated with the 24 hour dietary diversity score implying that we might not rely on this measurement to assess the nutritional status.

\section{Keywords}

Dietary Diversity Score, Malnutrition, Mid-Upper Arm Circumference, Pregnant Women, Antenatal

\section{Introduction}

Nutritional status of a woman is important for positive pregnancy outcome [1] [2]. The nutritional status can be assessed using different parameters, one of them being the mid-upper arm circumference (MUAC) [2] [3] [4], a simple measurement used to evaluate the nutritional status of an individual [5] [6] and serves as a surrogate of the body mass in pregnant women [7]. The MUAC is one of the most effective ways of determining short term change in the nutritional status of an identified population [8]. Biochemically nutritional status measurements in low-income countries are often too expensive to be used consistently; thus there is need to use anthropometric measurements such as MUAC to determine the nutritional status of individuals [9]. The MUAC, on the other hand can be influenced by the dietary diversity score (DDS). The DDS is a qualitative measure of food consumption reflecting an individual's access to different food items and therefore is a proxy indicator of the nutritional status [10]. The DDS is one of the easiest and effective ways of predicting under nutrition [11] [12]. The 24-hour dietary recall is a subjective report method of collecting data [13] where the individual has to recall what they have consumed in the last 24 hours.

There are different methods of conducting the survey, but the commonest one is a qualitative aspect where in-depth interviews are conducted by a trained interviewer or by self-reporting. Low intake of micronutrients caused by poor diet is one of the commonest causes of under nutrition [14]. However, a single 24-hour dietary recall is not enough to make adequate conclusions about an individual's food intake and nutrient adequacy [15], because it is based on evaluating a day's intake. To evaluate the effect of nutrient intake, other than using the DDS, it is necessary to consider the MUAC, which is an important tool in the assessment of the nutritional status [16]. The MUAC is measured as the circumference of the right hand from the mid-point between the olecranon process (tip of the shoulder) and the acromion (tip of the elbow) [17]. Generally, a MUAC of between $22 \mathrm{~cm}$ and $23 \mathrm{~cm}$ indicates undernourishment [18] though this may vary from country to country [19]. 
The cut-off point for MUAC varies from one area to another. A study conducted in China considered a cut-off point of $22 \mathrm{~cm}$ MUAC for the malnourished category of women; $7.3 \%$ of pregnant women were found to be malnourished but when the MUAC cut off was raised to $23 \mathrm{~cm}$ the number of malnourished women rose to $14.7 \%$ [20]. This indicates that the phenomenon of having women in the malnourished category is not limited to Zambia. In this study, the following MUAC cut-off points were considered; $\leq 26.3 \mathrm{~cm}$ and below were considered to be malnourished and $\geq 26.4$ as normal [16].

Dietary intake is an important indicator of pregnancy outcome [21] [22]. Research has shown that poor diet quality can be associated with a negative cognitive function of an individual [23] thus stressing the need for dietary intake assessment methods. In most African countries, most of the women rely on starchy staples, vegetables and seasonal fruits for their diet and these are plenty in rainy season [24] [25]. In Zambia, the staple food is nsima, a thick porridge made out of maize meal or cassava in some areas and it is eaten with meat, beans and green leafy vegetables.

The primary objective of this paper was to assess whether the DDS and MUAC can be used to assess the nutritional status in pregnant women attending the first antenatal visit. In Zambia, there are limited data on methods of nutritional assessment of women during antenatal care especially on the use of MUAC thus the reason why the investigator decided to embark on this study. Knowledge on the use of anthropometric measurements in pregnancy such as MUAC would enable adjustments on quality of care after diagnosing the nutritional status.

\section{Methods}

\subsection{Study Setting}

The study was conducted at Chilenje level I Hospital in a township located in Lusaka city, Lusaka province. Chilenje is a medium income residential area [26] [27]. However, the study area comprises a mixture of community members in the medium and low-income category. This has a bearing on the type of diet that the women were exposed to, that is, ranging from adequate to inadequate. The majority of the occupants were self-employed with a few in formal employment. Most of the people who were self-employed were engaged in small-scale businesses. Most of the families were able to meet their basic needs. Some people consumed a lot of alcohol and drug abuse was prevalent in the area. During the study period, there was relatively a good amount of food available as the rainy period in Zambia is usually accompanied by fresh fruits and vegetables. The study was undertaken between December 2017 and June 2018 when most of the food items were readily available and relatively affordable.

\subsection{Study Design, Population and Sampling}

The study design was cross-sectional and employed quantitative data collection 
and analysis methods to investigate dietary patterns of individual women in the previous 24 hours before the survey. The study population comprised pregnant women attending antenatal care for the first time in their current pregnancy. Random sampling method was employed to select the Health facility and a study sample of 299 respondents was systematically selected. Women aged between 15 and 49 years of age with singleton pregnancy were eligible for the study. Women who had multiple pregnancy, on medication such as Zinc and those who had a blood transfusion three (3) months before the study were excluded.

\subsection{Data Collection and Analysis}

Data were collected using an interview schedule and analysed using the quantitative method. The women were asked to mention the food items, which they consumed in the last 24 hours of the survey. The data on DDS were categorized in nine food groups [10], scored as 0 if not eaten and 1 if a specific food item was consumed, regardless of the number of times within 24 hours for instance, consuming all the 9 food stuffs resulted in a maximum score of 9 . The following were the food items: 1) Starchy staples such as maize and cassava 2) dark green leafy vegetables 3) fruits and vegetables rich in vitamin A like pawpaw 4) fruits and vegetables without vitamin A like guavas and cucumbers 5) organ meat 6) meat and fish 7) eggs 8) legumes, nuts and seeds and 9) milk and milk products [13].

In this study, MUAC tape recommended by the Food and Agricultural Commission was used (UNICEF Supply Division). Women who had a cut off of MUAC $\leq 26.3 \mathrm{~cm}$ and below were considered malnourished and those $\geq 26.4$ as well nourished [16]. The median and interquartile range was used to compare the demographic characteristics and the women's DDS where data was not normally distributed.

Each variable was tested for normality before determining the correlation test to use. Log transformation was used to normalize dependent variables which were continuous so as to meet the assumptions of linear regression. Spearman's Correlation was used to determine the relationship between two continuous variables where data was not normally distributed and Pearson's correlation where data was normally distributed. Simple linear regression was used to determine the relationship between MUAC and the independent variables. All variables with $\mathrm{p}$ values of 0.25 and below in the univariate analysis were considered in the adjusted regression. All statistical analyses were done using STATA version 13 (Stata Corp, College Station, Texas, USA). A p value of $<0.05$ was considered statistically significant for all statistical tests.

\subsection{Ethical Approval}

The University of Zambia Biomedical Research Ethics Committee approved the study (UNZABREC Ref: 007-11-17). The study was conducted in accordance 
with the Helsinki declaration. Participants signed an informed written consent and were free to withdraw from the study whenever they wished to do so. Written informed consent was obtained from guardians or parents of participants who were below 16 years of age.

\section{Results and Discussion}

The results are described under three sections, the demographic characteristics of the participants, regression coefficient between MUAC and the independent variables and predictors of MUAC.

Demographic characteristics of the participants

Information on the demographic characteristics is shown in Table 1.

Table 1. Baseline characteristics of study participants $(n=296)$.

\begin{tabular}{|c|c|c|}
\hline Variable & Category & Frequency (\%) \\
\hline \multirow[t]{3}{*}{ Age (Years) } & $13-19$ & 8 \\
\hline & $20-24$ & 27 \\
\hline & $25-44$ & 65 \\
\hline \multirow[t]{2}{*}{ Educational level } & Primary & 19 \\
\hline & Secondary/tertiary & 81 \\
\hline \multirow{3}{*}{ Income } & Low & 82 \\
\hline & Medium & 6 \\
\hline & High & 12 \\
\hline \multirow[t]{2}{*}{ Employment status } & Yes & 34 \\
\hline & No & 66 \\
\hline \multirow[t]{3}{*}{ DDS } & Low $(0-3)$ & 44 \\
\hline & Medium (4 - 5 ) & 31 \\
\hline & High (6 - 9) & 25 \\
\hline \multirow[t]{2}{*}{ MUAC (cms) } & Low $(0-26.3)$ & 25 \\
\hline & Normal (26.4 - 50) & 75 \\
\hline \multirow[t]{3}{*}{ Trimester } & First & 11 \\
\hline & Second & 80 \\
\hline & Third & 10 \\
\hline \multirow[t]{2}{*}{ Marital status } & Married & 78 \\
\hline & Single & 22 \\
\hline \multirow[t]{2}{*}{ Alcohol intake } & Yes & 20 \\
\hline & No & 81 \\
\hline HIV status & Reactive & 82 \\
\hline Non-reactive & & \\
\hline
\end{tabular}

DDS $=$ Dietary Diversity Score; MUAC = Mid-Upper Arm Circumference. 
A total of 299 participants were interviewed. The majority of the participants, $65 \%$ were in the age group between 25 to 50 years. The youngest participant was aged 13 and the oldest, 44 years [28]. The median age was 27 years. Most of the women, $80 \%$ booked in the second trimester whereas only $11 \%$ (30) booked in the first trimester.

With regards to marital status, $78 \%$ of the study participants were married. The majority of these women, $81 \%(241)$ had attained at least secondary or tertiary education. Less than half, 34\% (100) of the women were in formal employment. At the time of the study (June 2018), 1 Zambian Kwacha (ZMW) was equivalent to USD 12. The lowest household income was USD 1.3 and most of the women $82 \%$ (131) had a household income of less than USD 1.90 per day. The lowest household income was USD 8.3 whereas the highest was USD 4167 with the median income of USD 12.5 .

Slightly below half, 44\% (104) of these women had a low DDS of 0 to 3 with 203 (75\%) having a mid-upper arm circumference between 26.4 and $50 \mathrm{~cm}$. Among all the women, only one smoked.

Our study attempted to use 24 hours DDS which is a proxy measure of nutrition and whether it can correlate with MUAC in pregnant women attending the first antenatal visit among women. The majority of the women booking in the second trimester are similar to a study conducted by Nsibu and others in the Democratic Republic of Congo where the majority of antenatal women reported to the clinic after the first trimester [29]. Reporting to the antenatal clinic in the first trimester is important as ailments can be detected in good time and treated before affecting the foetus [30]. Women in this study might have shunned early antenatal booking as most of them feel that pregnancy is a natural phenomenon and does not require much intervention by the hospital authorities [31].

The number of women who had a low DDS in this study suggests undernutrition though this is higher than the magnitude of the problem reported in Ethiopia and Kenya respectively [32] [33] (28.6\% and 31.7\%). It is possible that the observed differences with the two studies could be due to different MUAC cut-off points that were used or Body mass index (BMI) difference among regions. However, in our study, we did not calculate the BMI of the women. This is because it is difficult to calculate the BMI in pregnancy unless the woman knows the pre-pregnancy weight.

Regression co-efficient between MUAC and the independent variables (Table 2)

In this study, nutritional status was described by assessing the mid-upper arm circumference with the independent variables. For every unit increase in age, there was a $0.91 \mathrm{~cm}$ increase in the MUAC (95\% CI; 0.81 - 1.00) although this was not statistically significant. Moving from low to medium income the mid-upper arm circumference increased by 2.09 units but not statistically significant (95\% CI; 0.69 - 6.31). Moving from medium to high income the mid-upper arm circumference increased by $1.02 \mathrm{cms}$ but not statistically significant $(0.16-6.61)$. 
Table 2. Regression co-efficient between MUAC and the independent variables.

\begin{tabular}{|c|c|c|c|c|c|c|}
\hline \multirow[b]{2}{*}{ Variable } & \multicolumn{3}{|c|}{ MUAC $\geq 26.4$ (unadjusted) } & \multicolumn{3}{|c|}{ MUAC $\geq 26.4$ (adjusted) } \\
\hline & OR & $(95 \% \mathrm{CI})$ & p-value & $\mathrm{aOR}$ & $(95 \% \mathrm{CI})$ & p-value \\
\hline Age (yrs) & 0.88 & $0.83-0.93$ & $<0.001$ & 0.91 & $0.81-1.00$ & 0.072 \\
\hline \multicolumn{7}{|l|}{ Income } \\
\hline -Low & Ref. & & & & & \\
\hline -Medium & 0.76 & $0.34-1.71$ & 0.513 & 2.09 & $0.69-6.31$ & 0.190 \\
\hline -High & 0.40 & $0.11-1.41$ & 0.154 & 1.02 & $0.16-6.61$ & 0.985 \\
\hline \multicolumn{7}{|l|}{ Employed } \\
\hline No & Ref. & & & & & \\
\hline Yes & 0.47 & $0.25-0.90$ & 0.022 & 0.97 & $0.35-2.74$ & 0.804 \\
\hline \multicolumn{7}{|l|}{$\begin{array}{l}\text { Educational } \\
\text { status }\end{array}$} \\
\hline -Primary & Ref. & & & & & \\
\hline -Secondary & 1.26 & $0.62-2.59$ & 0.526 & 1.15 & $0.38-3.48$ & 0.804 \\
\hline -Tertiary & 0.58 & $0.24-1.42$ & 0.234 & 1.27 & $0.26-6.23$ & 0.768 \\
\hline \multicolumn{7}{|l|}{ Married } \\
\hline -Yes & Ref. & & & & & \\
\hline -No & 0.27 & $1.37-4.62$ & 0.003 & 1.54 & $0.46-5.10$ & 0.483 \\
\hline Parity & 0.71 & $0.56-0.89$ & 0.003 & 1.21 & $0.74-1.98$ & 0.445 \\
\hline Weight & 0.87 & $0.83-0.91$ & $<0.001$ & 0.88 & $0.74-0.93$ & $<0.001$ \\
\hline
\end{tabular}

aOR adjusted odds ratio; OR odds ratio; CI confidence interval; DDS-Dietary Diversity Score. $\mathrm{R}^{2}=$ $0.2955 \%$ on average, thus about $2 \%$ of the total variation in the MUAC can be explained by variations in the independent variables such as age, household income, occupation, and weight. Only body weight was statistically significant. The other variables did not have a significant influence on the MUAC.

For education status, moving from primary to secondary level of education, MUAC increased by $1.15 \mathrm{cms}$ (95\% CI; $0.38-3.48)$ and $1.27 \mathrm{cms}$ moving from primary to tertiary education (95\% CI; $0.26-6.23$ ) but both were not statistically significant. Moving from being married to being single the MUAC increased by $1.54 \mathrm{cms}(95 \% \mathrm{CI} ; 0.46-5.10)$ but not statistically significant. For every unit increase in the number of children MUAC increased by $1.21 \mathrm{cms}$ (95\% CI; 0.74 1.98 ) but not statistically significant. For every unit increase in weight there was a 0.88 unit increase in MUAC (95\% CI; $0.74-1.98)$ and this was statistically significant.

In our study, the DDS was negatively correlated with the MUAC. Similarly, Ghosh and his colleagues in their study, found that having a high DDS did not have a positive increase on the MUAC [34]. In this study, the DDS could have been negatively correlated with MUAC as the 24-hour dietary recall may not give a true reflection of the daily food intake of the participants. The positive correlation between age and MUAC is in keeping with a study among pregnant women conducted by Fakier et al., in the Metro West area of Cape Town [35]. A 
cross-sectional study in Bangladesh in both male and female adults showed that there was a positive linear correlation between MUAC and the Body Mass Index (BMI) [36] which justifies the use of anthropometric measurements in nutritional assessment. The BMI is difficult to calculate in pregnancy as it requires calculation of the pre-pregnancy weight which very few women can remember [37].

Being single and poor was independently associated with lower MUAC at first antenatal visit. Other studies have shown that being poor, single and parity of more than three are associated with lower MUAC [38] in keeping with this study. However, one of the differences between the two studies was that we did not find associations between MUAC and lower education but in the other studies associations were reported. On the other hand, the United States Agency for International Development (USAID) reported that high fertility and unwanted pregnancy contributed to decreased nutritional status in women [32].

In this study, the median age was 27 years which is higher than what is reported in other studies [39]. In our study we did not get information about age at first marriage but other studies have shown that women who were married or cohabiting were less likely to be under nourished [38]. Although in our study there was no association between alcohol and lower MUAC, some studies have reported that alcohol consumption is associated with under nutrition and lower MUAC [40]. This was probably due to the lower number of women who reported that they consumed alcohol (20\%) compared to other studies where there is almost equal number of women who consume alcohol to those who do not [41] [42]. We did not determine the association between MUAC and smoking because of the insufficient numbers (1) of participants that smoked in this study. However others have reported that women who smoke during pregnancy were more likely to have lower MUAC and lower baby birth weight compared to non-smokers [43].

Other studies have reported that HIV positive status was independently associated with lower MUAC [44]. One would speculate that it could be due to the independent effect of HIV on the immune system which is likely to be observed especially in immune compromised states. Surprisingly, in this study there was no association between HIV and lower MUAC suggesting that maybe it was not an immune compromised group although we did not check for CD4 or viral load to confirm our claims.

Predictors of Mid-Upper Arm Circumference

Results for the predictors of MUAC are shown in Table 3. Consumption of green leafy vegetables resulted in a 0.58-unit increase in MUAC (95\% CI; 0.31 1.07) but not statistically significant. Consumption of vitamin A rich fruits and vegetables resulted in a $1.03 \mathrm{~cm}$ increase in MUAC (95\% CI; $0.54-1.93$ ) but not statistically significant. Consumption of organ meat resulted in a $0.21 \mathrm{~cm}$ increase in MUAC and was statistically significant (95\% CI; 0.04 - 0.97). Consumption of fish and meat resulted in a $1.17 \mathrm{~cm}(95 \% \mathrm{CI} ; 0.61-2.21)$ increase in 
Table 3. Logistic regression between mid-upper arm circumference and dietary diversity sore.

\begin{tabular}{lllllll}
\hline \multirow{2}{*}{ Variables } & \multicolumn{2}{l}{ MUAC $\geq 26.4$ (unadjusted) } & \multicolumn{3}{l}{ MUAC $\geq 26.4$} \\
\cline { 2 - 7 } & OR & $(95 \% \mathrm{CI})$ & p value & aOR & $(95 \%$ CI) & p value \\
\hline Starchy staples & & - & & - & & \\
Dark green leafy vegetables & 0.06 & $(0.32-0.98)$ & 0.04 & 0.58 & $(0.31-1.07)$ & 0.08 \\
Vitamin A fruits and vegetables & 0.89 & $(0.51-1.55)$ & 0.67 & 1.03 & $(0.54-1.93)$ & 0.92 \\
Organ meat & 0.18 & $(0.04-0.81)$ & 0.02 & 0.21 & $(0.04-0.97)$ & 0.04 \\
Meat and fish & - & - & - & 1.17 & $(0.61-2.21)$ & 0.63 \\
Other fruits & 0.57 & $(0.19-1.75)$ & 0.33 & 0.61 & $(0.19-1.91)$ & 0.39 \\
Eggs & 0.74 & $(0.42-0.34)$ & 0.33 & 0.80 & $(0.42-1.53)$ & 0.50 \\
Legumes and nuts & 0.97 & $(0.56-1.71)$ & 0.94 & - & - & - \\
Milk & 1.39 & $(0.77-2.53)$ & 0.27 & 1.77 & $(0.91-3.42)$ & 0.09 \\
\hline
\end{tabular}

$\mathrm{aOR}=$ adjusted odds ratio; $\mathrm{OR}=$ odds ratio; $\mathrm{CI}=$ confidence interval.

MUAC but this was not statistically significant. Consumption of other fruits not rich in vitamin A was associated with a $0.61 \mathrm{~cm}$ increase in MUAC (95\% CI; 0.19 - 1.91) but not statistically significant. Consumption of eggs was associated with a $0.80 \mathrm{~cm}$ increase in MUAC (95\% CI; 0.42 - 1.53) but this was not statistically significant. Consumption of milk was associated with a $1.77 \mathrm{~cm}$ increase in MUAC (95\% CI; 0.91 - 3.42) but this was not statistically significant.

In our study, the majority of the women consumed starchy staples as part of their diet. This could be due to the fact that Zambia's main staple food is maize [45]. This is confirmed by the Science Brief on Bio fortification which explains that most of the African countries rely on maize as a staple food [46]. Eating of dark green leafy vegetables was significant in the univariate analysis but not in the multivariate one. These findings have some similarities with the National Food and Nutrition Commission where it was discovered that most of the pregnant women took green leafy vegetables as part of their diet most of the time [47]. Consumption of the vegetables in the Zambian diet is very common as they are cheap and most of the people cannot afford a varied diet. Eating vegetables however is an advantage as one is protected from cancer [48] [49]. On the contrary, a study conducted in northern Ghana revealed that pregnant women who were of a low socio-economic status ate less vegetables compared to those who were of a higher socio-economic status. In the same study, there was a strong negative association between the consumption of animal products in the previous 24 hours and socio-economic status [50].

A cross-sectional descriptive study aimed at evaluating dietary habits among pregnant women in Turkey revealed that most of the foods consumed were fruits and vegetables with a decrease in the consumption of tea and red meat [22]. Pregnant women from a low socio-economic class were two times more likely to be undernourished compared to pregnant women from food secure households [32]. In the same study, pregnant women and their husbands who had low levels 
of education were more likely to be undernourished compared to those who had higher levels of education showing that the educational status can have a bearing on the nutritional status of individuals.

A study aimed at assessing the consumption pattern and dietary practices of women in Nigeria revealed high consumption of cereals and grains, in particular rice, the mostly consumed fruits were oranges and green leafy vegetables, whereas fish, meat and eggs were eaten on a daily basis [51]. The study limitations include the fact that most of the participants were from a middle income background, however, non-adjusted and adjusted analysis was performed to factor in for the confounders. Additionally, it is possible that there was recall bias on the type of food consumed the previous day but women were given time to think about the activities that took place.

\section{Conclusion}

In this study, it was discovered that $44 \%$ of the women were in the lowest DDS category, which indicates inadequate nutrient intake, $31 \%$ in the medium category and $24 \%$ in the high intake. This implies that most of the women are unable to take adequate nutrients. These results might be attributed to the fact that most of the women (40\%) were in the lower income category as the income may have a bearing on the quality and quantity of food [52] [53]. In this study, the DDS was negatively correlated with MUAC which is unusual as we expect the MUAC to be higher under normal circumstances. This suggests that the food consumed in the previous 24 hours before the study did not have an impact on the MUAC. Despite most of the women being in the lower DDS category, most of them (75\%) were in the higher MUAC category meaning that their nutritional status was good. However, the study findings might not be generalized to the rural areas of Zambia where dietary patterns differ from the urban population.

\section{Acknowledgements}

Authors wish to thank the Norwegian Programme for Capacity Development in Higher Education and Research for Development (NORHED) for providing sponsorship, the Tropical Gastroenterology and Nutritional Group (TROPGAN) for providing analysis of micronutrients, EDCTP through TESA II project (Grant number 1051 TESAII EDCTP-RegNe) that facilitated the "data management boot camp", contributed to the development of this manuscript and the Southern African Consortium for Research Excellence (SACORE) for technical assistance, Chilenje Hospital team for helping in data collection and the participants.

\section{Conflicts of Interest}

The authors declare that they have no competing interests.

\section{References}

[1] Lim, Z., Wong, J., Lim, P., Soon, L. and Sciences C. (2018) Knowledge of Nutrition 
during Pregnancy and Associated Factors among Antenatal Mothers. International Journal of Public Health and Clinical Sciences (IJPHCS), 5, 117-128.

[2] Oliver, E., Grimshaw, K., Schoemaker, A., Keil, T., Mcbride, D., Sprikkelman, A., et al. (2014) Dietary Habits and Supplement Use in Relation to National Pregnancy Recommendations: Data from the Europrevall Birth Cohort. Maternal and Child Health Journal, 18, 2408-2425. https://doi.org/10.1007/s10995-014-1480-5

[3] Bhattacharya, A., Pal, B., Mukherjee, S. and Roy, S.K. (2019) Assessment of Nutritional Status Using Anthropometric Variables by Multivariate Analysis. BMC Public Health, 19, Article No. 1045. https://doi.org/10.1186/s12889-019-7372-2

[4] VanTonder, E., Mace, L., Steenkamp, L., Tydeman-Edwards, R., Gerber, K. and Friskin, D. (2019) Mid-Upper Arm Circumference (MUAC) as a Feasible Tool in Detecting Adult Malnutrition. South African Journal of Clinical Nutrition, 32, 93-98. https://doi.org/10.1080/16070658.2018.1484622

[5] Benítez Brito, N., Suárez Llanos, J.P., Fuentes Ferrer, M., Oliva García, J.G., Delgado Brito, I., Pereyra-García Castro, F., et al. (2016) Relationship between Mid-Upper Arm Circumference and Body Mass Index in Inpatients. PLoS ONE, 11, e0160480. https://doi.org/10.1371/journal.pone.0160480

[6] Debnath, S., Mondal, N. and Sen, J. (2017) Use of Upper Arm Anthropometry, Upper Arm Muscle Area-by-Height (UAMAH) and Midupper-Arm-Circumference (MUAC)-for-Height as Indicators of Body Composition and Nutritional Status among Children. Anthropological Review, 80, 85-102.

https://doi.org/10.1515/anre-2017-0004

[7] Fakier, A., Petro, G. and Fawcus, S. (2017) Mid-Upper Arm Circumference: A Surrogate for Body Mass Index in Pregnant Women. South African Medical Journal, 107, 606-610. https://doi.org/10.7196/SAMJ.2017.v107i7.12255

[8] Frison, S. (2017) Middle-Upper Arm Circumference for Nutritional Surveillance in Crisis-Affected Populations: Development of a Method. London School of Hygiene \& Tropical Medicine, London.

[9] Kulathinal, S., Freese, R., Korkalo, L., Ismael, C. and Mutanen, M. (2016) Mid-Upper Arm Circumference Is Associated with Biochemically Determined Nutritional Status Indicators among Adolescent Girls in Central Mozambique. Nutrition Research, 36, 835-844. https://doi.org/10.1016/j.nutres.2016.04.007

[10] Ngala, S. (2015) Evaluation of Dietary Diversity Scores to Assess Nutrient Adequacy among Rural Kenyan Women. Wageningen University, Wageningen.

[11] Zhao, W., Yu, K., Tan, S., Zheng, Y., Zhao, A., Wang, P., et al. (2017) Dietary Diversity Scores: An Indicator of Micronutrient Inadequacy Instead of Obesity for Chinese Children. BMC Public Health, 17, Article No. 440.

https://doi.org/10.1186/s12889-017-4381-x

[12] Caswell, B.L., Talegawkar, S.A., Siamusantu, W., West Jr., K.P. and Palmer, A.C. (2018) A 10-Food Group Dietary Diversity Score Outperforms a 7-Food Group Score in Characterizing Seasonal Variability and Micronutrient Adequacy in Rural Zambian Children. The Journal of Nutrition, 148, 131-139. https://doi.org/10.1093/jn/nxx011

[13] Gina Kennedy, B.T. and Dop, M.C. (2012) Guidelines for Measuring Household and Individual Dietary Diversity. Food and Agriculture Organization of the United Nations, Rome.

[14] Shim, J.-S., Oh, K. and Kim, H.C. (2014) Dietary Assessment Methods in Epidemiologic Studies. Epidemiology and Health, 36, e2014009.

https://doi.org/10.4178/epih/e2014009 
[15] FAO (2018) Dietary Assessment. Food and Agriculture Organization of the United Nations, Rome.

[16] Tang, A.M., Chung, M., Dong, K., Terrin, N., Edmonds, A., Assefa, N., et al. (2016) Determining a Global Mid-Upper Arm Circumference Cutoff to Assess Malnutrition in Pregnant Women. FHI 360/Food and Nutrition Technical Assistance III Project (FANTA), Washington DC.

[17] Yallamraju, S.R., Mehrotra, R., Sinha, A., Gattumeedhi, S.R., Gupta, A. and Khadse, S.V. (2014) Use of Mid Upper Arm Circumference for Evaluation of Nutritional Status of OSMF Patients. Journal of International Society of Preventive \& Community Dentistry, 4, 122-125. https://doi.org/10.4103/2231-0762.146217

[18] Ververs, M.-T., Antierens, A., Sackl, A., Staderini, N. and Captier, V. (2013) Which Anthropometric Indicators Identify a Pregnant Woman as Acutely Malnourished and Predict Adverse Birth Outcomes in the Humanitarian Context? PLoS Currents, 5.

[19] Alvarez, J.L., Dent, N., Browne, L., Myatt, M. and Briend, A. (2018) Mid-Upper Arm Circumference (MUAC) Shows Strong Geographical Variations in Children with Edema: Results from 2277 Surveys in 55 Countries. Archives of Public Health, 76, Article No. 58. https://doi.org/10.1186/s13690-018-0290-4

[20] Gao, H., Stiller, C.K., Scherbaum, V., Biesalski, H.K., Wang, Q., Hormann, E., et al. (2013) Dietary Intake and Food Habits of Pregnant Women Residing in Urban and Rural Areas of Deyang City, Sichuan Province, China. Nutrients, 5, 2933-2954. https://doi.org/10.3390/nu5082933

[21] Ramlal, R.T., Tembo, M., King, C.C., Ellington, S., Soko, A., Chigwenembe, M., et al. (2015) Dietary Patterns and Maternal Anthropometry in HIV-Infected, Pregnant Malawian Women. Nutrients, 7, 584-594. https://doi.org/10.3390/nu7010584

[22] Eren, N.Ş., Şencan, İ, Aksoy, H., Koç, E.M., Kasım, İ., Kahveci, R., Samur, G. and Özkara, A. (2015) Evaluation of Dietary Habit during Pregnancy. Turkish Journal of Obstetrics and Gynecology, 12, 89-95. https://doi.org/10.4274/tjod.79923

[23] Wright, R.S., Waldstein, S.R., Kuczmarski, M.F., Pohlig, R.T., Gerassimakis, C.S., Gaynor, B., et al. (2016) Diet Quality and Cognitive Function in an Urban Sample: Findings from the Healthy Aging in Neighborhoods of Diversity across the Life Span (HandLS) Study. Public Health Nutrition, 20, 92-101. https://doi.org/10.1017/S1368980016001361

[24] Kiboi, W. (2016) Dietary Diversity, Nutrient Intake and Nutritional Status among Pregnant Women in Laikipia County, Kenya. Department of Food, Nutrition and Dietetics, Kenyatta University, Kenya.

[25] Kinyua, L.W. (2013) Association of Nutrition Knowledge and Attitude with Dietary Practices and Nutritional Status of Female Undergraduate Students Attending University Colleges within Nairobi Metropolis. Unpublished Doctoral Dissertation, the University of Nairobi, Nairobi.

[26] Owino, V., Amadi, B., Sinkala, M., Filteau, S. and Tomkins, A. (2008) Complementary Feeding Practices and Nutrient Intake from Habitual Complementary Foods of Infants and Children Aged 6-18 Months Old in Lusaka, Zambia. African Journal of Food, Agriculture, Nutrition and Development, 8, 28-47. https://doi.org/10.4314/ajfand.v8i1.19176

[27] Simatele, D., Binns, T. and Simatele, M. (2012) Sustaining Livelihoods under a Changing Climate: The Case of Urban Agriculture in Lusaka, Zambia. Journal of Environmental Planning and Management, 55, 1175-1197.

https://doi.org/10.1080/09640568.2011.637688 
[28] Central Statistical Office (CSO) [Zambia], Ministry of Health ( $\mathrm{MOH}$ ) [Zambia] and ICF International (2014) Zambia Demographic and Health Survey 2013-14. Central Statistical Office, Ministry of Health, and ICF International, Rockville, Maryland.

[29] Nsibu, C.N., Manianga, C., Kapanga, S., Mona, E., Pululu, P., Aloni, M.N.J.O., et al. (2016) Determinants of Antenatal Care Attendance among Pregnant Women Living in Endemic Malaria Settings: Experience from the Democratic Republic of Congo. Obstetrics and Gynecology International, 2016, Article ID: 5423413. https://doi.org/10.1155/2016/5423413

[30] Qin, J.-B., Feng, T.-J., Yang, T.-B., Hong, F.-C., Lan, L.-N., Zhang, C.-L., et al. (2014) Risk Factors for Congenital Syphilis and Adverse Pregnancy Outcomes in offspring of Women with Syphilis in Shenzhen, China: A Prospective Nested Case-Control Study, 41, 13-23. https://doi.org/10.1097/OLQ.0000000000000062

[31] Qureshi, R.N., Sheikh, S., Khowaja, A.R., Hoodbhoy, Z., Zaidi, S., Sawchuck, D., et al. (2016) Health Care Seeking Behaviours in Pregnancy in Rural Sindh, Pakistan: A Qualitative Study. Reproductive Health, 13, Article No. 34. https://doi.org/10.1186/s12978-016-0140-1

[32] Nigatu, M., Gebrehiwot, T.T. and Gemeda, D.H. (2018) Household Food Insecurity, Low Dietary Diversity, and Early Marriage Were Predictors for Undernutrition among Pregnant Women Residing in Gambella, Ethiopia. Advances in Public Health, 2018, Article ID: 1350195. https://doi.org/10.1155/2018/1350195

[33] Rana, M.J. and Goli, S. (2017) Family Planning and Its Association with Nutritional Status of Women: Investigation in Select South Asian Countries. Indian Journal of Human Development, 11, 56-75. https://doi.org/10.1155/2018/1350195

[34] Ghosh, S., Spielman, K., Kershaw, M., Ayele, K., Kidane, Y., Zillmer, K., et al. (2019) Nutrition-Specific and Nutrition-Sensitive Factors Associated with Mid-Upper Arm Circumference as a Measure of Nutritional Status in Pregnant Ethiopian Women: Implications for Programming in the First 1000 Days. PLoS ONE, 14, e0214358. https://doi.org/10.1371/journal.pone.0214358

[35] Grellety, E. and Golden, M.H. (2016) Weight-for-Height and Mid-Upper-Arm Circumference Should Be Used Independently to Diagnose Acute Malnutrition: Policy Implications. BMC Nutrition, 2, Article No. 10. https://doi.org/10.1186/s40795-016-0049-7

[36] Sultana, T., Karim, M.N., Ahmed, T. and Hossain, M.I. (2015) Assessment of Under Nutrition of Bangladeshi Adults Using Anthropometry: Can Body Mass Index Be Replaced by Mid-Upper-Arm-Circumference? PLOS ONE, 10, e0121456. https://doi.org/10.1371/journal.pone.0121456

[37] Russell, A., Gillespie, S., Satya, S. and Gaudet, L.M. (2013) Assessing the Accuracy of Pregnant Women in Recalling Pre-Pregnancy Weight and Gestational Weight Gain. Journal of Obstetrics and Gynaecology Canada, 35, 802-809. https://doi.org/10.1016/S1701-2163(15)30836-7

[38] Mtumwa, A.H., Paul, E. and Vuai, S.A.H. (2016) Determinants of Undernutrition among Women of Reproductive Age in Tanzania Mainland. South African Journal of Clinical Nutrition, 29, 75-81. https://doi.org/10.1080/16070658.2016.1216509

[39] Feyissa, T.R. and Genemo, G.A. (2014) Determinants of Institutional Delivery among Childbearing Age Women in Western Ethiopia, 2013: Unmatched Case Control Study. PLOS ONE, 9, e97194. https://doi.org/10.1371/journal.pone.0097194

[40] Nunes, G., Santos, C.A., Barosa, R., Fonseca, C., Barata, A.T. and Fonseca, J. (2017) Outcome and Nutritional Assessment of Chronic Liver Disease Patients Using Anthropometry and Subjective Global Assessment. Arquivos de Gastroenterologia, 54, 
225-231. https://doi.org/10.1590/s0004-2803.201700000-28

[41] Watts, R., Linke, S., Murray, E. and Barker, C. (2015) Calling The Shots: Young Professional Women's Relationship with Alcohol. Feminism \& Psychology, 25, 219-234. https://doi.org/10.1177/0959353515571670

[42] Colell, E., Sánchez-Niubò, A. and Domingo-Salvany, A. (2013) Sex Differences in the Cumulative Incidence of Substance Use by Birth Cohort. International Journal of Drug Policy, 24, 319-325. https://doi.org/10.1016/j.drugpo.2012.09.006

[43] Zheng, W., Suzuki, K., Tanaka, T., Kohama, M., Yamagata, Z. and the Okinawa Child Health Study Group (2016) Association between Maternal Smoking during Pregnancy and Low Birthweight: Effects by Maternal Age. PLoS ONE, 11, e0146241. https://doi.org/10.1371/journal.pone.0146241

[44] Wilkinson, A.L., Pedersen, S.H., Urassa, M., Michael, D., Todd, J., Kinung'hi, S., et al. (2015) Associations between Gestational Anthropometry, Maternal HIV, and Fetal and Early Infancy Growth in a Prospective Rural/Semi-Rural Tanzanian Cohort, 2012-13. BMC Pregnancy and Childbirth, 15, Article No. 277.

https://doi.org/10.1186/s12884-015-0718-6

[45] Faostat, F. (2012) Disponível. http://faostat.fao.org/

[46] Menkir, A., Palacios-Rojas, N., Alamu, O., et al. (2018) Vitamin A-Biofortified Maize: Exploiting Native Genetic Variation for Nutrient Enrichment. Cimmyt, Iita, Embrapa, HarvestPlus, and Crop Trust Bonn, Germany.

[47] The Infant \& Young Child Nutrition (IYCN) and Project and the National Food and Nutrition Commission (2010) Qualitative Assessment of Maternal Nutrition Practices in Zambia. National Food and Nutrition Commission, Lusaka.

[48] Norat, T., Scoccianti, C., Boutron-Ruault, M.C., Anderson, A., Berrino, F., Cecchini, M., et al. (2015) European Code against Cancer 4th Edition: Diet and Cancer. Cancer Epidemiology, 39, S56-S66. https://doi.org/10.1016/j.canep.2014.12.016

[49] Schwingshackl, L., Schwedhelm, C., Galbete, C. and Hoffmann, G. (2017) Adherence to Mediterranean Diet and Risk of Cancer: An Updated Systematic Review and Meta-Analysis. Nutrients, 9, 1063. https://doi.org/10.3390/nu9101063

[50] Saaka, M., Oladele, J., Larbi, A. and Hoeschle-Zeledon, I. (2017) Household Food Insecurity, Coping Strategies, and Nutritional Status of Pregnant Women in Rural Areas of Northern Ghana. Food Science \& Nutrition, 5, 1154-1162. https://doi.org/10.1002/fsn3.506

[51] Ademuyiwa, M. and Sanni, S. (2013) Consumption Pattern and Dietary Practices of Pregnant Women in Odeda Local Government Area of Ogun State. International Journal of Biological, Biomolecular, Agricultural, Food and Biotechnological Engineering, 7, 11-15.

[52] Corfe, S.J.S.M.F. (2018) What Are the Barriers to Eating Healthily in the UK.

[53] Anderson, P.M., Butcher, K.F.J.C.O.B. and June P.P.P. (2016) The Relationships among SNAP Benefits, Grocery Spending, Diet Quality, and the Adequacy of Low-Income Families' Resources. Center for Budget and Policy Priorities Policy Futures, Washington DC, 14. 